\title{
Numerical-Experimental Updating Identification of Elastic Behavior of a Composite Plate Using New Multi-Objective Optimization Procedure
}

\author{
Samir Ghanmi ${ }^{*}$, Sofiane Bouajila ${ }^{2}$, Mohamed Guedri $^{3}$ \\ ${ }^{1}$ Nabeul Preparatory Engineering Institute-IPEIN, Nabeul, Tunisia; ${ }^{2}$ Higher Institute of Technological Studies of Nabeul, Nabeul, \\ Tunisia; ${ }^{3}$ Higher School of Science and Technology of Tunis, Tunis, Tunisia. \\ Email: ${ }^{3}$ samir.ghanmi@ipein.rnu.tn
}

Received August $6^{\text {th }}, 2012$; revised September $2^{\text {nd }}, 2012$; accepted September $8^{\text {th }}, 2012$

\begin{abstract}
This work focuses on the updating-based identification of the three-dimensional orthotropic elastic behavior of a thin carbon fiber reinforced plastic multilayer composite plate. This consists in identifying the engineering constants that minimize the relative deviations between the first eight experimental and three-dimensional finite element frequencies of the vibrating free plate. For this purpose, a multi-objective optimization procedure is applied; it exploits a Particle Swarm Optimizer algorithm (PSO) that is coupled to a metamodeling by the new response surfaces method procedure (NRSMP); the latter is based on numerical design experiments. The conducted sensitivity analyses indicate that the four engineering constants of the two-dimensional elasticity are the most influent.
\end{abstract}

Keywords: Composites; Finite Element Experimental Designs; Multi-Objective Optimization; Numerical-Experimental Updating Identification; Response Surfaces

\section{Introduction}

During the last three decades, composite materials are widely used in modern structures for high performance and reliability because of their high strength, specific stiffness, light weight and adjustable properties. However, before using this type of material with confidence in industrial applications such as marine, automotive or aerospace structural components, a thorough characterization of the constituent material properties is needed. Because of the number and the inherent variability of the constitutive properties of composite materials, the experimental characterization is quite cumbersome and requires a large number of specimens to be tested.

To circumvent this lack, an effective procedure consists in using mixed numerical-experimental methods which constitute powerful tools for estimating unknown constitutive coefficients in a numerical model of a composite structure from static and/or dynamic experimental data collected on the real structure.

Starting from the measurement of quantities such as the natural frequencies and mode shapes, these methods allow, by comparing numerical and experimental observations, the progressive refinement of the estimated material properties in the corresponding numerical model. In this

\footnotetext{
"Corresponding author.
}

domain, dynamic mixed techniques have gained in importance owing to their simplicity and efficiency $[1,2]$.

In this work, a new mixed numerical-experimental identification method based on the use of a multi-objective optimization procedure. It exploits a Particle Swarm Optimizer algorithm (PSO) [3-5] that is coupled to a meta-modeling by the new response surfaces method procedure (NRSMP) based on Droesbeke et al. and Myers et al. works [6,7]; this procedure is applied to estimate unknown constitutive parameters from a numerical model of a composite plate and its experimental data of dynamic tests.

This method is based on a technique for minimizing the differences between the first eight numerical frequencies of the composite structure and the corresponding experimental modal values [8].

In our case, the constitutive parameters that can be identified are three in-plane Young's moduli $E_{1}, E_{2}$ and $E_{3}$ and the in-plane and transverse shear moduli $G_{12}, G_{13}$ and $G_{23}$, the Poisson's ratios $v_{12}, v_{13}$ and $v_{23}$.

This optimization procedure is preceded by a sensitivity analysis using ANOVA [7] in order to eliminate noninfluent parameters on the behavior of the composite structure.

The multi-objective problem to be solved is of eight cost functions and nine design parameters which are: $E_{1}$, 
$E_{2}, E_{3}, G_{12}, G_{13}, G_{23}, v_{12}, v_{13}$ and $v_{23}$.

\section{Elastic Behavior of Composites}

The macroscopic mechanical properties of composite materials depend on their microstructure. The micro structural organization that is generally not regular, often leads to anisotropy at the macroscopic level. This anisotropy can be explained mechanically as a dependency of the elastic response vis-à-vis the direction of stress.

However, for most of the industrial composites, this behavior is often orthotropic, described by the stressstrain relationship:

$$
\sigma_{i}=C_{i j} \varepsilon_{j}
$$

where the terms $\sigma_{i}$ and $\varepsilon_{j}$ represent components of the stress and strain tensors, respectively. $C_{i j}$ is the matrix of elastic coefficients. The matrix of elastic coefficients for an orthotropic material is written as follows:

$$
C_{i j}=\left[\begin{array}{cccccc}
C_{11} & C_{12} & C_{13} & & & \\
& C_{22} & C_{23} & & & \\
& & C_{33} & & & \\
& & & C_{44} & & \\
& S y m & & & C_{55} & \\
& & & & & C_{66}
\end{array}\right]
$$

There are twelve non-zero components of which nine are independent. (2.1) can be rewritten in terms of the compliance matrix, $S_{i j}$ :

$$
\varepsilon_{j}=S_{i j} \sigma_{i}
$$

The compliance matrix for an orthotropic material expressed in terms of elastic properties such as Young's modulus, shear modulus and Poisson's ratio and is:

$$
C_{i j}^{-1}=S_{i j}=\left[\begin{array}{cccccc}
\frac{1}{E_{1}} & -\frac{v_{21}}{E_{1}} & -\frac{v_{31}}{E_{1}} & & & \\
-\frac{v_{12}}{E_{1}} & \frac{1}{E_{2}} & -\frac{v_{32}}{E_{2}} & & & \\
-\frac{v_{13}}{E_{1}} & -\frac{v_{23}}{E_{2}} & \frac{1}{E_{3}} & & & \\
& & & \frac{1}{G_{23}} & & \\
& & & & \frac{1}{G_{13}} & \\
& & & & & \frac{1}{G_{12}}
\end{array}\right]
$$

where $E_{i}$ are the Young's moduli in the $i$ direction, $v_{i j}$ are Poisson's ratio for strain in the $j$ direction with stress applied in the $i$ direction, and $G_{i j}$ are the shear moduli in the $i-j$ plane $(i, j=1,2$ or 3$)$. Since the matrix of elastic coefficients and the compliance matrix are symmetric, hence

$$
\frac{v_{i j}}{E_{i}}=\frac{v_{j i}}{E_{j}}
$$

\section{Problem Data}

We studied a composite plate that was cut by the manufacturer from a large panel of length $1.56 \mathrm{~m}$, width $1.06 \mathrm{~m}$, $4.16 \mathrm{~mm}$ in thickness and density of $1512 \mathrm{~kg} / \mathrm{m}^{3}$. The panel material is of IMS/977-2 type (Figure 1).

The panel consists of 16 layers with the stacking symmetric sequence of [90/45/0/-45 $]_{25}$ : its elastic properties are provided by the manufacturer (modulus in $\mathrm{GPa}$ ):

$$
\begin{aligned}
& E_{1}=160 ; E_{2}=8.6 ; G_{12}=4.85 ; v_{12}=0.32 \\
& E_{3}=E_{2} ; G_{13}=G_{12} ; v_{13}=v_{12} \\
& G_{23}=\frac{2}{3} G_{12}=3.2 ; v_{23}=0.4(\text { estimate })
\end{aligned}
$$

The plate was provided by the manufacturer with the following nominal dimensions : $300 \mathrm{~mm}$ in length (y axis) and $200 \mathrm{~mm}$ wide (x axis); however, the checks in the laboratory, provides [8] a width of $200.3 \mathrm{~mm}$, an average thickness of $4.2 \mathrm{~mm}$ and a density of $1521 \mathrm{~kg} / \mathrm{m}^{3}$. It is these data that were used to make a first simulation of the free plate configuration using 3D solid elements Quadratic hexahedral ABAQUS ${ }^{\circledR}$ element C3D20R: to 17303 nodes, 2400 elements: 51909 dof.

Figure 2 shows the first eight modes of the plate to be studied.

The measured frequencies [8] and simulated, are provided in Table 1 for the first eight modes of vibration free. We note that numerical frequencies greatly overestimate

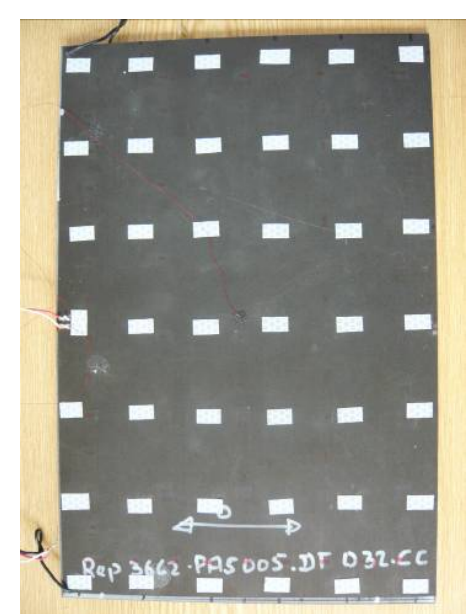

(a)

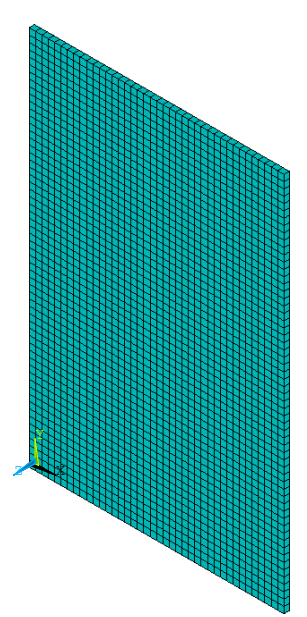

(b)
Figure 1. Tested plate (a) and 3D FE model (b). (a) Tested composite plate [8]; (b) 3D FE model. 
Table 1. Experimental frequencies $(\mathrm{Hz})$ and simulated by $\mathrm{FE}$ of the composite plate.

\begin{tabular}{cccccccccc}
\hline Modes & 1 & 2 & 3 & 4 & 5 & 6 & 7 & 8 \\
\hline Exp. Freq. [8] & 229.3 & 310.9 & 554.6 & 581 & 724.7 & 889.1 & 1068.4 & 1163.7 \\
FE Freq. with Equation (3.1) & 246.24 & 345.43 & 596.68 & 608.49 & 775.66 & 972.84 & 1142.7 & 1264.6 \\
Error (\%) & 7.39 & 11.11 & 7.59 & 4.73 & 7.03 & 9.42 & 6.95 & 8.67 \\
FE Freq. with Equation (3.2) & 234.86 & 319.51 & 568.54 & 594.66 & 740.27 & 912.01 & 1095.3 & 1194.3 \\
Error (\%) & 2.42 & 2.76 & 2.51 & 2.35 & 2.14 & 2.57 & 2.51 & 2.62 \\
\hline
\end{tabular}

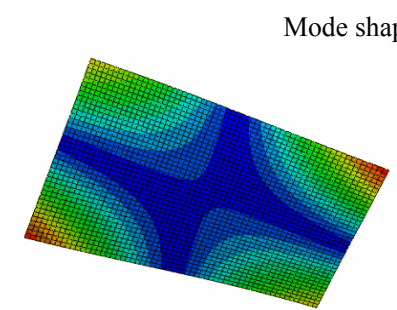

Mode 1

Mode 3

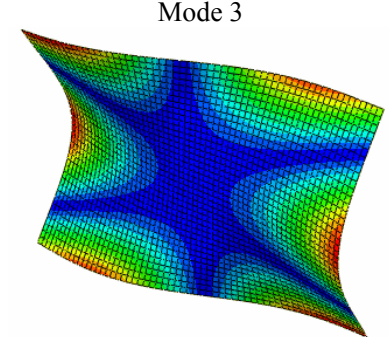

Mode 5

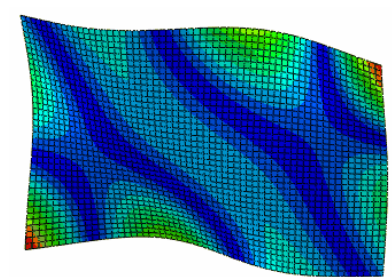

Mode 7

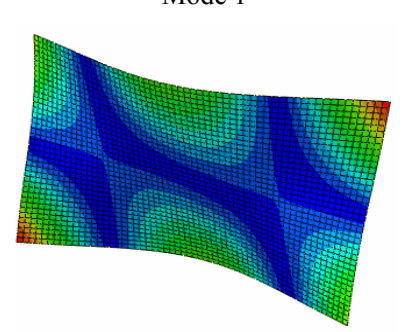

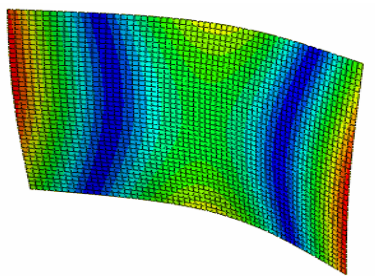

Mode 2

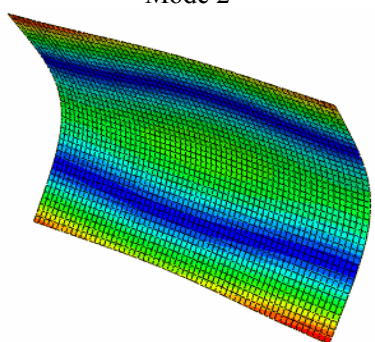

Mode 4

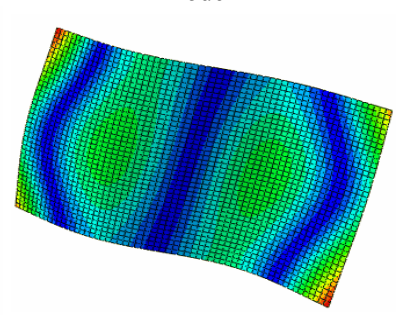

Mode 6

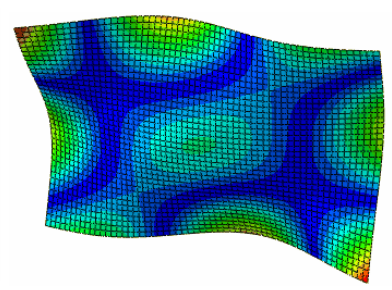

Mode 8
Figure 2. First eight modes of the composite plate.

those measured, suggesting that elastic properties of the plate, given by manufacturer, overestimate those of the plate tested. Measurements on another plate of the same nominal size, also cut by the manufacturer from the same large panel, provided an average thickness of $4.13 \mathrm{~mm}$ and a density of $1547 \mathrm{~kg} / \mathrm{m}^{3}$, these new data confirm that the material of panel is not uniform and identification of its elastic properties is required; this plate gave the following constants (modulus in $\mathrm{GPa}$ ) [9]:

$$
\begin{aligned}
& E_{1}=129.8 ; E_{2}=10.9 ; G_{12}=5.7 ; v_{12}=0.37 ; \\
& G_{13}=4.4 ; G_{23}=3.2 ; E_{3}=E_{2} ; v_{13}=v_{12} ; v_{23}=0.55
\end{aligned}
$$

These results confirm that the design parameters provided by the manufacturer are generally largely overestimated or underestimated.

The obtained frequencies are shown in Table 1. We denote this time that correlation between experiment and calculation is satisfactory; the residual errors indicate that they are probably due to differences in the dimensions (width and thickness) and densities of the two plates.

It is therefore proposed, in what follows, to identify elastic constants of this plate by updating the experimental frequencies [8] and simulated by ABAQUS ${ }^{\circledR} 3 \mathrm{D} F E$ using a proposed multi-objective optimization procedure retaining the data (Equation (7)) as initial values and we take as variations on the parameters $\pm 20 \%$ around their mean values.

\section{Identification by Multi-Objective Optimization Procedure}

Figure 3 presents the proposed methodology for the identification of mechanical properties of the composite plate (Figure 1).

Its three main steps are detailed in the following.

\subsection{Sensitivity Analyze}

Sensitivity analysis studies the "sensitivity" of the outputs of a system to changes in the parameters, inputs or initial conditions which are often poorly known. Its goal is to show the effects of changing parameter values and their interactive effects and then eliminate those that are not significant on the output.

Sensitivity analysis is based on the use of Numerical Design of Experimental (NDOE) which enables designers to determine simultaneously the individual and interactive effects of many factors that could affect the output results in any design. NDOE also provides a full insight of interaction between design elements; therefore, it helps 


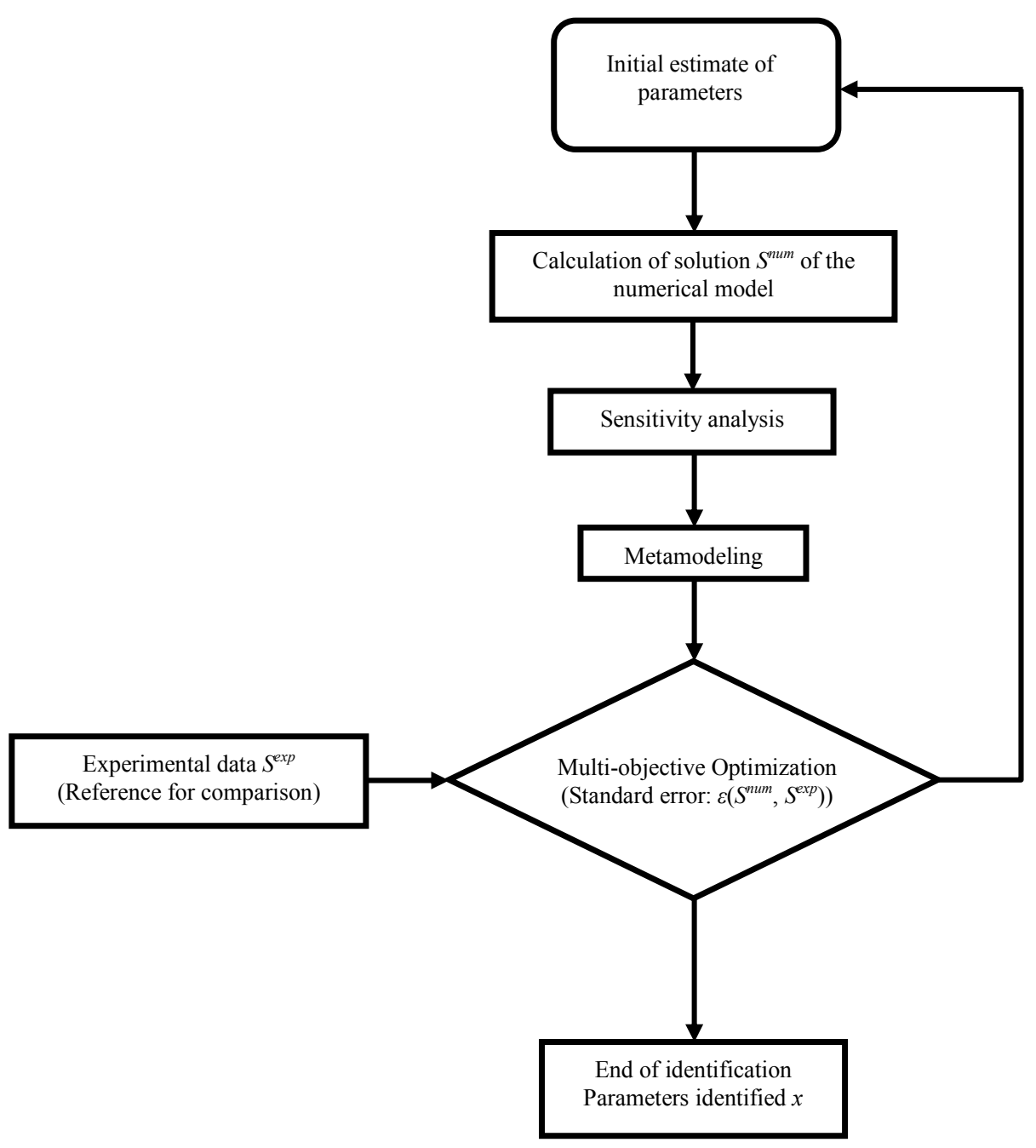

Figure 3. Proposed procedure of identification.

turn any standard design into a robust one.

\subsection{Metmodeling by New Response Surface Method Procedure}

The metamodeling is based on the use of NRSMP which is based on the use of numerical design of experiments. The aim is to represent each frequency by a polynomial which will be easier to use in the optimization phase.

The linear models used here are second-order regression models (4.1). A RSM for $k$ input variables can be stated as:

$$
\hat{y}=\beta_{0}+\sum_{i=1}^{k} \beta_{i} \cdot x_{i}+\sum_{\substack{i=1, j=2 \\ i<j}}^{k} \beta_{i j} \cdot x_{i} \cdot x_{j}+\sum_{i=1}^{k} \beta_{i i} \cdot x_{i}^{2}+\cdots+\varepsilon
$$

This model requires at least $2 n+1+n(n-1) / 2$ simulations to be completely defined. Where $y$ is the response variable, $x$ are the input variables, and the $\beta$ 's are the coefficients to be estimated.

Equation (4.1) may be written in matrix notation as:

$$
Y=X \beta+\varepsilon
$$

where

$$
\begin{gathered}
Y=\left[\begin{array}{c}
y_{1} \\
y_{2} \\
\vdots \\
y_{n}
\end{array}\right] ; X=\left[\begin{array}{ccccc}
1 & x_{11} & x_{12} & \cdots & x_{1 k} \\
1 & x_{21} & x_{22} & \cdots & x_{2 k} \\
\vdots & \vdots & \vdots & & \vdots \\
1 & x_{n 1} & x_{n 2} & \cdots & x_{n k}
\end{array}\right] ; \\
\beta=\left[\begin{array}{c}
\beta_{1} \\
\beta_{2} \\
\vdots \\
\beta_{n}
\end{array}\right] \text { and } \varepsilon=\left[\begin{array}{c}
\varepsilon_{1} \\
\varepsilon_{2} \\
\vdots \\
\varepsilon_{n}
\end{array}\right]
\end{gathered}
$$

In general, $Y$ is an $n \times 1$ vector of the observations, $X$ is an $n \times p$ the matrix of the levels of the independent variables, $\beta$ is a $p \times 1$ vector of the regression coefficients, and $\varepsilon$ is an $n \times 1$ vector of random errors. 
Use of only one configuration (a metamodel) which considers the real response appears in sufficient owing to the fact that it can be statistically far from the most precise polynomial which can be considered as the exact response. To surmount this disadvantage, we propose a method which aims to obtain the met model (polynomial) nearest to the reference. The components stages of the method suggested for obtaining the adequate met model are summarized as follows:

1) Number of design parameters $(n)$;

2) Complete factorial design;

3) Random generation of " $k$ " polynomials;

4) Statistical and frequency criteria satisfied or go to step 2;

5) Calculation of the polynomial average $P_{m}$ : where $n_{\text {rest }}$ is the number of the noted remaining polynomials $P_{\text {rest }}$ :

$$
P_{m}=\frac{\sum_{i=1}^{n_{\text {rest }}} P_{\text {rest }}}{n_{\text {rest }}}
$$

Note: The designer generates randomly several polynomials of the function to estimate. Each polynomial obtained is evaluated statistically (MSE criterion for example). The set of polynomials that did not meet the criteria for evaluation constitute: $P_{\text {rest }}$.

6) Generation of several samples of size " $p$ " by Latin hypercube method (LHC);

7) Acceptable error of prediction $\varepsilon_{m}$ or go to step 2.

The statistical criteria of selection are:

- The error MSE which is defined by :

$$
M S E=\frac{\sum_{i=1}^{m}\left(\hat{y}_{i}-\bar{y}_{i}\right)^{2}}{m \cdot \sigma_{\text {exact }}^{2}}
$$

where $\sigma_{\text {exact }}$ is the standard deviation of exact response $y_{i}$.

In practice, to satisfy of a process of the 6 sigma criterion an error MSE should not exceed value 0.09028 [10].

- The coefficient of determination $R^{2}$ is defined by:

$$
R^{2}=\frac{1-\sum_{i=1}^{m}\left(\hat{y}_{i}-\bar{y}_{i}\right)^{2}}{\sum_{i=1}^{m}\left(y_{i}-\bar{y}_{i}\right)^{2}}
$$

where $y_{i}$ is the exact response; $\hat{y}_{i}$ is the estimated response and $\bar{y}_{i}$ is the mean value of the exact response. This coefficient must be as close as possible to value $1(0$ $\left.<R^{2}<1\right)[6,7]$.

\subsection{Multi-Objective Particle Swarm Optimization}

In mechanical engineering, the optimization problems are often multi-objective. The cost functions are complex (multimodal, non-convex, etc.) and there are generally conflicts between them. Therefore, it is necessary to choose a multi-objective optimization strategy which can lead to the best alternatives among several.

Generally, a multi-objective optimization problem is expressed by:

$$
\left\{\begin{array}{c}
\min F(x)=\left(f_{1}(x), f_{2}(x), \cdots, f_{n}(x)\right)^{T} \\
x \in S
\end{array}\right.
$$

where $f_{1}(x), f_{2}(x), \cdots, f_{n}(x)$ are cost functions, $x=\left(x_{1}, x_{2}, \cdots, x_{n}\right)^{T}$ is the vector of $n$ optimization parameters, $S \in R^{n}$ represent the set of realizable solutions and $F(x)$ is the vector of the functions to be optimized.

In this study, we exploit the multi-objective Particle Swarm Optimization algorithm (PSO). This is justified by several reasons. In fact, Particle swarm optimization (PSO) is a stochastic optimization method often used to solve complex engineering where the solution may be represented as a hyper dimensional vector (such as $3 \mathrm{D}$ space). The method's strength lies in its simplicity, being easy to code and requiring few algorithm parameters to define convergence behavior.

Similar to evolutionary optimization methods, PSO is a derivative-free, population-based global search algorithm. PSO uses a population of solutions, called particles, which fly through the search space with directed velocity vectors to find better solutions. These velocity vectors have stochastic components and are dynamically adjusted based on historical and inter-particle information $[11,12]$.

\section{Identification of Elastic Behavior of the Composite Plate}

The proposed identification procedure, which based of multi-objective optimization, is applied here to this composite plate described above. Multi-objective problem to be solved is in eight cost functions (5.1).

$$
\left\{\begin{array}{c}
\min F(x)=\varepsilon_{i}(x)=\left|\frac{\hat{f}_{i}(x)-f_{i}^{\exp }}{f_{i}^{\exp }}\right| ; i=1, \cdots, 8 \\
x \in\left[E_{1}, E_{2}, E_{3}, G_{12}, G_{23}, v_{12}, v_{13}, v_{23}\right]
\end{array}\right.
$$

\subsection{Sensitivity Analysis}

A $2^{k}$ factorial design of experiments was used. The use of nine design parameters (factors), each at two levels leading to a total of $2^{9}=512$ numerical experiments to be performed. These parameters vary in fields of $\pm 20 \%$ around their mean values given by (3.2). To make this analysis, an ANOVA was performed for a significance level of $5 \%$, that is to say, for a confidence level of $95 \%$.

There is a significant variation of $E_{1}$, which is the most influential factor on the behavior of the composite plate 
and a slight variation on each of the parameters $E_{2}, E_{3}, G_{12}$, $G_{23}, v_{12}, v_{13}$ and $v_{23}$ (Figure 4). The transverse parameters $E_{3}, G_{13}, G_{23}, v_{13}$ and $v_{23}$ have no significant effect. Three in-plane parameters, $E_{1}, E_{2}, G_{12}$, are most influential, especially $E_{1}$. From this analysis we find that the $E_{1}, E_{2}$, $G_{12}, v_{12}$ factors.

\subsection{Metamodeling by Response Surface Method}

In this phase, we consider the results of sensitivity analysis above. The new problem is thus to four design parameters (significant parameters), each at two levels leading to a total of $2^{4}=16$ numerical experiments to be performed. These parameters vary in fields of $\pm 20 \%$ around their mean values given by (3.2).

The non significant factors are frozen in their mean values.

The metamodels for each of the frequencies of the first eight vibration modes of free composite plate are given by the equations in Table 2 .

Each polynomial is statistically validated by a factor of determination $R^{2}$, which should be as close as possible to 1, and an MSE (Mean Square Error), which must be less than 0.09028 [10].

Note that the metamodels (polynomials) of Table 2 meet all these criteria. This allows us to use these polynomials in the optimization phase.

\subsection{Multi-Objective Optimization Procedure}

The multi-objective optimization is achieved by coupling the PSO algorithm to the metamodel. Taking into account of the results of sensitivity analysis, the new multiobjective problem to be solved is:

$$
\left\{\begin{array}{c}
\min F(x)=\varepsilon_{i}(x)=\left|\frac{\hat{f}_{i}(x)-f_{i}^{\exp }}{f_{i}^{\exp }}\right| ; i=1, \cdots, 8 \\
x \in\left[E_{1}, E_{2}, G_{12}, v_{12}\right]
\end{array}\right.
$$

This problem replaces the multi-objective problem of Equation (5.1).

The application of multi-objective optimization procedure, with coupling PSO algorithm with metamodels (cost functions) of Table 2, led to the optimal values of design parameters that are given (after rounding) in Tables 3 and 4 .

Table 3 shows the values of design parameters provided by the manufacturer and those given by optimization. These values are compared each time by the mean values of design parameters. The variations of parameters (Table 3) show that the manufacturer has underestimated the parameters $E_{2}, E_{3}, G_{12}, v_{12}, v_{13}$ and $v_{23}$ and overestimated parameters $E_{2}$ and $G_{23}$.

Table 4 presents the eight frequencies from this optimization and residual errors (both rounded). Note that these errors are very satisfactory.

\section{Conclusions}

The updating-based identification of the three-dimensional orthotropic elastic behavior of a thin carbon fiber reinforced plastic multilayer composite plate is presented.

This consists in identifying the engineering constants that minimize the relative deviations between the first eight experimental and three-dimensional finite element frequencies of the vibrating free plate.

For this purpose, a new multi-objective optimization procedure is applied; it exploits a Particle Swarm Optimization algorithm that is coupled to a meta-modeling by new response surfaces method procedure; the latter is based on numerical design experiments. The conducted sensitivity analyses indicate that the four engineering constants of two-dimensional elasticity are the most influent.

The proposed identification procedure confirms that the design parameters provided by manufacturer are gen-

Table 2. Estimated frequencies.

\begin{tabular}{cccc}
\hline Freq. & Polynomials & $R^{2}$ & MSE $\left(10^{-7}\right)$ \\
\hline$f_{1}$ & $\hat{f}_{1}=234,5325+17,5412 x_{1}+1.1412 x_{2}+4.5950 x_{3}-0.5600 x_{4}$ & 0.9995 & $4.22 \times 10^{-4}$ \\
$f_{2}$ & $\hat{f}_{2}=318,3456+28,7331 x_{1}+1.2069 x_{2}+2.0594 x_{3}+0.0631 x_{4}$ & 0.9999 & $5.28 \times 10^{-5}$ \\
$f_{3}$ & $\hat{f}_{3}=567,9181+44,3219 x_{1}+3.3556 x_{2}+8.5244 x_{3}-0.4769 x_{4}$ & 0.9997 & $2.56 \times 10^{-4}$ \\
$f_{4}$ & $\hat{f}_{4}=593,4081+47,9044 x_{1}+8.4056 x_{2}+3.1606 x_{3}+2.1481 x_{4}$ & 0.9997 & $3.26 \times 10^{-4}$ \\
$f_{5}$ & $\hat{f}_{5}=739,2506+58,8194 x_{1}+5.3531 x_{2}+9.1206 x_{3}-0.7331 x_{4}$ & 0.9998 & $2.07 \times 10^{-4}$ \\
$f_{6}$ & $\hat{f}_{6}=909,8025+80,4700 x_{1}+6.6500 x_{2}+3.2987 x_{3}+3.1963 x_{4}$ & 0.9998 & $1.75 \times 10^{-4}$ \\
$f_{7}$ & $\hat{f}_{7}=194.3+86.3 x_{1}+8.3 x_{2}+13 x_{3}+0.6 x_{4}$ & 0.9998 & $1.44 \times 10^{-4}$ \\
$f_{8}$ & $\hat{f}_{8}=1194.6+95.2 x_{1}+6.6 x_{2}+15.1 x_{3}-0.5 x_{4}$ & 0.9997 & $2.58 \times 10^{-4}$ \\
\hline
\end{tabular}


Numerical-Experimental Updating Identification of Elastic Behavior of a Composite

Plate Using New Multi-Objective Optimization Procedure

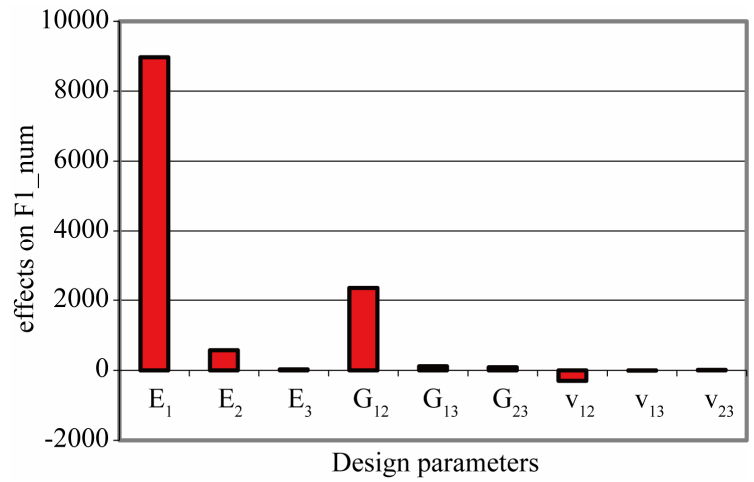

(a)

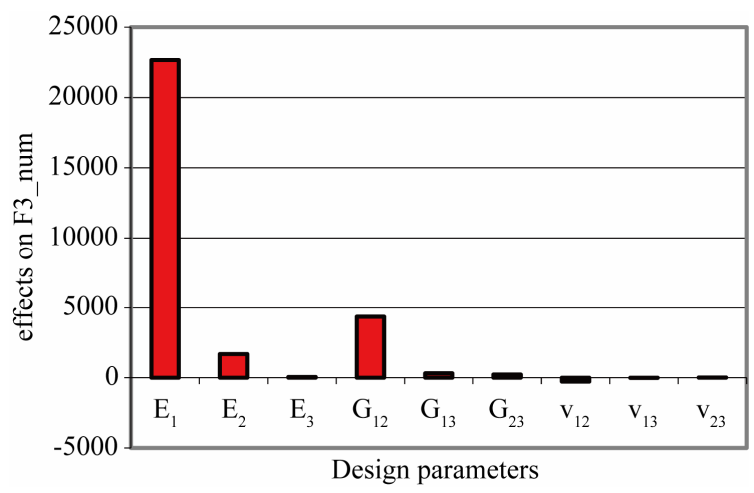

(c)

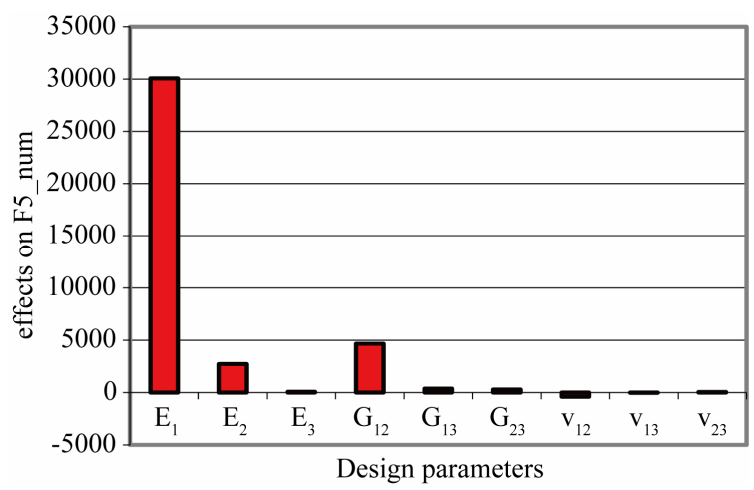

(e)

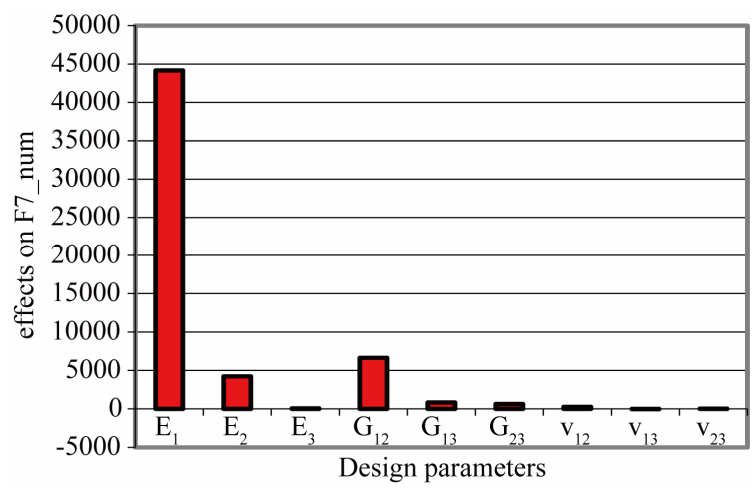

(g)

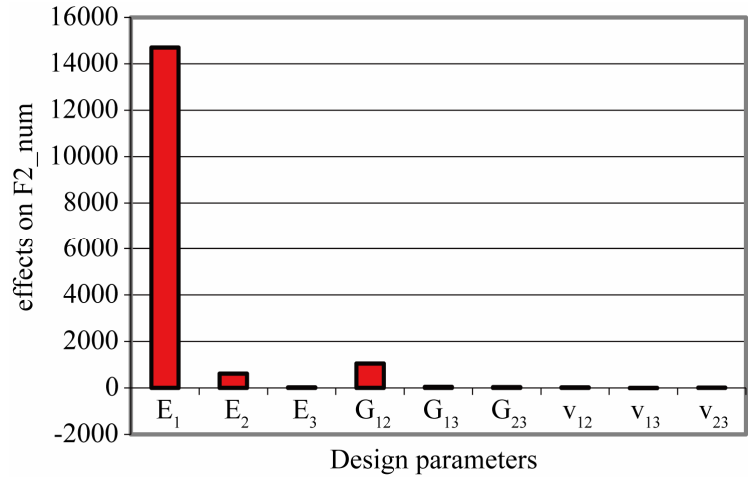

(b)

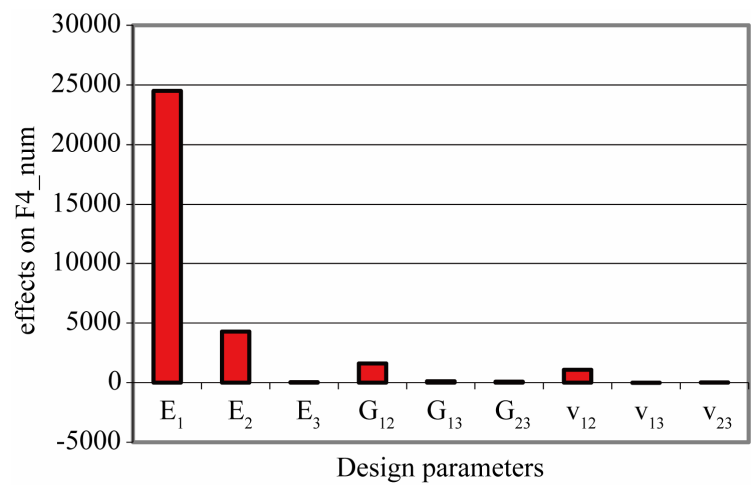

(d)

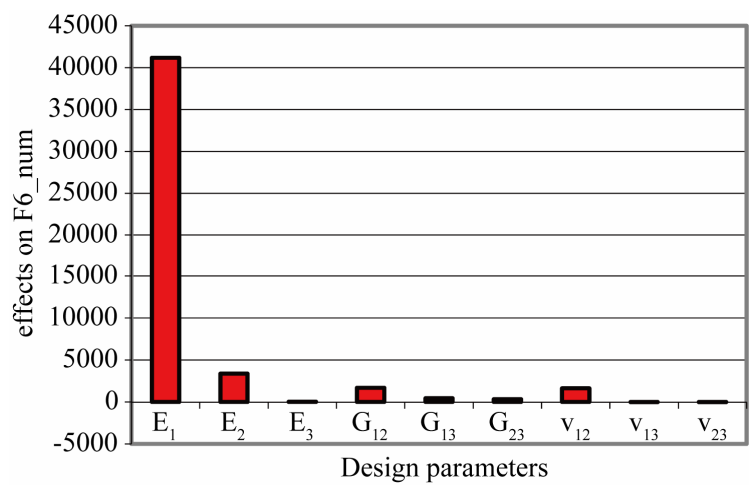

(f)

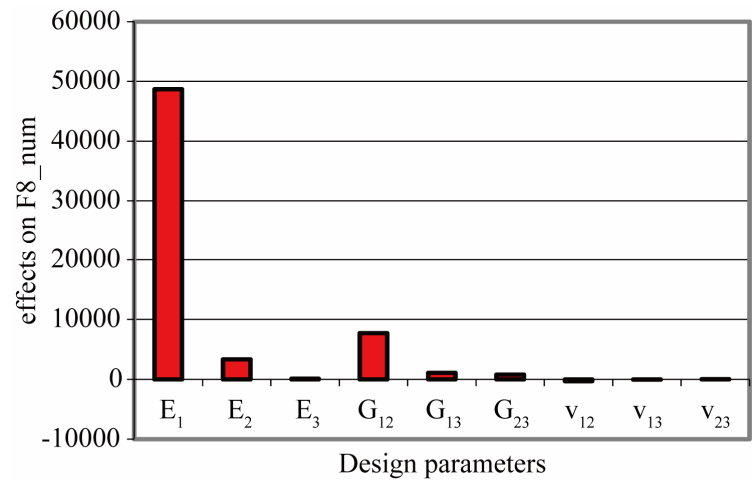

(h)

Figure 4. Effects of design parameters on eight eigenfrequencies. (a) Mode 1; (b) Mode 2; (c) Mode 3; (d) Mode 4; (e) Mode 5; (f) Mode 6; (g) Mode 7; (h) Mode 8. 
Table 3. Optimal values and variations of design parameters.

\begin{tabular}{cccccccccc}
\hline Parameters & $E_{1}(\mathrm{GPa})$ & $E_{2}(\mathrm{GPa})$ & $E_{3}(\mathrm{GPa})$ & $G_{12}(G P a)$ & $G_{13}(G P a)$ & $G_{23}(G P a)$ & $v_{12}$ & $v_{13}$ & $v_{23}$ \\
\hline Mean Values (M.M) (3.2) & 129.8 & 10.9 & 10.9 & 5.7 & 4.4 & 3.2 & 0.37 & 0.37 & 0.55 \\
Manufacturer (M) & 160 & 8.6 & 8.6 & 4.85 & 4.85 & 3.2 & 0.32 & 0.32 & 0.40 \\
Variation M/M.M (\%) & 23.27 & -21.10 & -21.10 & -14.91 & 10.28 & 0 & -13.51 & -13.51 & -6.27 \\
After Optimization (A.O) & 120.1 & 11.6 & 9.6 & 5.9 & 4.3 & 3.8 & 0.37 & 0.44 & 0.59 \\
Variation A.O/M.M (\%) & -7.45 & 6.42 & -11.93 & 3.50 & -2.27 & 18.75 & 0 & 18.92 & 7.27 \\
\hline
\end{tabular}

Table 4. Frequencies (Hz) after optimization and residual errors (\%).

\begin{tabular}{ccccccccc}
\hline Modes & 1 & 2 & 3 & 4 & 5 & 6 & 7 & 8 \\
\hline Exp. Freq. [8] & 229.3 & 310.9 & 554.6 & 581 & 724.7 & 889.1 & 1068.4 & 1163.7 \\
Opt. Freq. & 229.87 & 310.36 & 555.99 & 580.37 & 723.14 & 887.54 & 1071.2 & 1168.2 \\
Error (\%) & 0.25 & 0.17 & 0.25 & 0.11 & 0.21 & 0.17 & 0.26 & 0.39 \\
\hline
\end{tabular}

erally largely overestimated or underestimated. Therefore, before using mechanical structures like composite plates, it makes sense to correctly identify their mechanical properties to avoid any further risks, hence the interest of our proposed identification procedure.

\section{REFERENCES}

[1] J. Cugnoni, "Identification and Registration Modal Frequency of Shell Constitutive Properties of Composite Materials," Ph.D. Thesis, Federal Polytechnic School of Lausanne, 2005.

[2] M. Hamdi, S. Ghanmi, A. Benjeddou and R. Nasri, "Identification by Multi-Objective Optimization of ThreeDimensional Elastic Behavior of a Multilayer Composite," First International Symposium IMPACT, Djerba, 2010.

[3] M. Clerc and P. Siarry, "A New Metaheuristic for Difficult Optimization Method of Particle Swarm," Vol. 3-7, 2004.

[4] J. Kennedy and R. C. Eberhart, "Particle Swarm Optimization," Proceedings of the IEEE International Conference on Neural Networks, 1995, Vol. 4, pp. 1942-1948. doi:10.1109/ICNN.1995.488968

[5] J. F. Schutte, B. Koh, J. A. Reinbolt, R. T. Haftka, A. D. George and B. J. Fregly, "Evaluation of a Particle Swarm
Algorithm for Biomechanical Optimization," Journal of Biomechanical Engineering, Vol. 127, No. 3, 2005, pp. 465-474.

[6] J. J. Droesbeke, J. Fine and G. Saporta, "Design of Experiments: Applications in the Company," Paris, 1997.

[7] R. H. Myers and D. C. Montgomery, "Response Surface Methodology," Wiley, New York, 2002.

[8] G. Chevalier and A. Benjeddou, "Coupling Effective Electromechanical Piezoelectric Composite Structures: Experimental and Numerical Characterization," Journal of Composites and Advanced Materials, Vol. 19. No. 3, 2009, pp. 339-364.

[9] H. Friedmann, "Non-Destructive Parameter Estimation, in CASSEM D9-Test Report on Experimental Validation for Test/Model Correlation," March 2008, pp. 5-13.

[10] G. J. Battaglia and J. M. Maynard, "Mean Square Error: A Useful Tool for Statistical Process Management," AMP Journal of Technology, Vol. 34, 1996, pp. 1256-1260.

[11] A. A. Groenwold and P. C. Fourie, "The Particle Swarm Optimization in Size and Shape Optimization," Structural and Multidisciplinary Optimization, Vol. 23, 2002, pp. 259267. doi:10.1007/s00158-002-0188-0

[12] T. Cura, "Particle Swarm Optimization Approach to Portfolio Optimization," Nonlinear Analysis: Real World Applications, Vol. 10, No. 4, 2009, pp. 2394-2406. 